\title{
Weitere Untersuchungen zur Gruppenaxiomatik
}

\author{
Von Bengt Stolt
}

\section{§ 1. Einleitung}

In meiner Dissertation ${ }^{1}$ habe ich nebst vollständigen und unvollständigen Axiomensysteme auch einige unbestimmte Systeme aufgestellt. Der Zweck der vorliegenden Arbeit ist $u$. a. die Vollständigkeit von noch vier Systemen zu zeigen. Es wird auch gezeigt, dass die hergeleiteten Systeme irreduzibel sind.

Weiter zeige ich, wie es möglich ist, durch Einführung zweier neuen Axiome vollständige Systeme herzuleiten, die weniger Axiome als die in der Dissertation hergeleiteten Systeme enthalten.

Für Bezeichnungen und Hilfssätze wird an die eben angeführte Dissertation verwiesen. Es wird hervorgehoben, dass, wenn eines der Axiome $l \varepsilon . J, l v . J, r \varepsilon . J$ und $r v . J$ besteht, es zwei Elemente $c$ und $d$ gibt, die eine gewisse Verknüpfung erfüllen. Wenn mehrere von diesen Axiomen bestehen, werden wir annehmen, dass $c$ und $d$ in allen Verknüpfungen dieselben sind.

\section{§ 2. Das System $A, E, U, l E . i, l U . i, r \in . i, r u . i$}

In diesem Abschnitt wollen wir den folgenden Satz beweisen.

Satz 1. Das System A, E. U, lE.i,lU.i, re.i, rv.i ist vollständig.

Beweis: Der Annahme zufolge existiert ein $d$ mit $l E . i, l U . i, r \varepsilon . i$ und rv.i. Wegen Hilfssatz 2 im Abschnitt II bestehen $e d=d$ und $d e=d$, wo $e$ ein Element mit $l E . I$ und $l U . I$ ist.

Zunächst werden wir zeigen, dass $e$ auch die Eigenschaften $r \varepsilon . I$ und $r v . I$ hat. Wegen $r \varepsilon . i$ und $r v . i$ gibt es zu $d$ und einem gewissen $c$ genau ein $h^{\prime}$ mit $c h^{\prime}=d$, und wegen $l E . I$ und $l U . I$ bestehen $c^{\prime} c=e$ und $c_{\alpha} c^{\prime}=e$. Nun bilden wir

$$
(e c) h^{\prime}=e\left(c h^{\prime}\right)=e d=d,
$$

woraus nach $l U . i e c=c$ folgt. Ferner erhalten wir

und

$$
\left(c^{\prime} e\right) c=c^{\prime}(e c)=c^{\prime} c=e
$$

$$
\left(e c^{\prime}\right) c=e\left(c^{\prime} c\right)=e e=e,
$$

1 Stout, B.: Über Axiomensysteme, die eine abstrakte Gruppe bestimmen. Uppsala 1953. 


\section{B. SToLt, Weitere Untersuchungen zur Gruppenaxiomatik}

woraus $c^{\prime} e=c^{\prime}$ und $e c^{\prime}=c^{\prime}$ folgen. Wir bilden auch

$$
\left(c_{\alpha} e\right) c^{\prime}=c_{\alpha}\left(e c^{\prime}\right)=c_{\alpha} c^{\prime}=e,
$$

woraus $c_{\alpha} e=c_{\alpha}$ folgt, und

$$
c=e c=\left(c_{\alpha} c^{\prime}\right) c=c_{\alpha}\left(c^{\prime} c\right)=c_{\alpha} e=c_{\alpha},
$$

woraus $c_{\alpha}=c$ folgt. Folglich bestehen $c e=c$ und $c c^{\prime}=e$.

Um zu zeigen, dass auch rv.I besteht, nehmen wir an, dass es ein $c_{\alpha}^{\prime}$ mit $c c_{\alpha}^{\prime}=e$ gibt. Aus

und

$$
e\left(c_{\alpha}^{\prime} d\right)=\left(c c_{\alpha}^{\prime}\right) d=e d=d
$$

$$
c\left(c^{\prime} d\right)=\left(c c^{\prime}\right) d=e d=d
$$

folgen $c_{\alpha}^{\prime} d=h^{\prime}$ und $c^{\prime} d=h^{\prime}$.

Wegen $l E . I$ und $l U . I$ gilt $d^{\prime} d=e$. Dann erhalten wir

$$
\left(d d^{\prime}\right) d=d\left(d^{\prime} d\right)=d e=d,
$$

woraus $d d^{\prime}=e$ folgt. Wir bilden ferner

$$
h^{\prime} d^{\prime}=\left(c^{\prime} d\right) d^{\prime}=c^{\prime}\left(d d^{\prime}\right)=c^{\prime} e=c^{\prime},
$$

woraus $h^{\prime} d^{\prime}=c^{\prime}$, und

$$
c_{\alpha}^{\prime} e=c_{\alpha}^{\prime}\left(d d^{\prime}\right)=\left(c_{\alpha}^{\prime} d\right) d^{\prime}=h^{\prime} d^{\prime}=c^{\prime} .
$$

Dann gilt $c_{\alpha}^{\prime} e=c^{\prime}$. Schliesslich erhalten wir

$$
c_{\alpha}^{\prime} c=c_{\alpha}^{\prime}(e c)=\left(c_{\alpha}^{\prime} e\right) c=c^{\prime} c=e,
$$

und dann folgt $c_{\alpha}^{\prime} c=e$. Wegen $l U . I$ gilt $c_{\alpha}^{\prime}=c^{\prime}$, und folglich besteht rv.I.

Für ein beliebiges $a$ mit $a^{\prime} a=e$ gelten wegen Hilfssatz 2 im Abschnitt II $a a^{\prime}=e_{\alpha}$ und $e_{\alpha} e_{\alpha}=e_{\alpha}$. Wir werden nun zeigen, dass $e_{\alpha}$ gleich $e$ ist.

Wegen $l E . I$ und $l U . I$ gilt $e_{\alpha}^{\prime} e_{\alpha}=e$. Wir erhalten

$$
e e_{\alpha}=\left(e_{\alpha}^{\prime} e_{\alpha}\right) e_{\alpha}=e_{\alpha}^{\prime}\left(e_{\alpha} e_{\alpha}\right)=e_{\alpha}^{\prime} e_{\alpha}=e,
$$

woraus $e e_{\alpha}=e$ folgt.

Wegen

gilt $c e_{\alpha}=c$, und aus

$$
c e_{\alpha}=(c e) e_{\alpha}=c\left(e e_{\alpha}\right)=c e=c
$$

$$
c\left(e_{\alpha} c^{\prime}\right)=\left(c e_{\alpha}\right) c^{\prime}=c c^{\prime}=e
$$

folgt wegen $r v . I e_{\alpha} c^{\prime}=c^{\prime}$. Schliesslich bilden wir

$$
e_{\alpha} e=e_{\alpha}\left(c^{\prime} c\right)=\left(e_{\alpha} c^{\prime}\right) c=c^{\prime} c=e .
$$

Folglich gilt $e_{\alpha} e=e$. Wegen $l U . I$ gilt $e_{\alpha}=e$. Hiermit ist gezeigt, dass das Element $e$ auch die Eigenschaft $r E . I$ hat, and das System ist auf das vollständige System 7) im Abschnitt II zurückgeführt. 
ARKIV För MATEMATIK. Bd $3 \mathrm{nr} 5$

\section{§ 3. Das System $A, E, l E, U, l v . i, r \epsilon . i$, ru.i}

Die Vollständigkeit des im folgenden Satz aufgestellten Systems wurde bei der Disputation von Lennart Carleson gezeigt.

Satz 2. Das System $A, E, l E, U, l v . i, r \varepsilon . i, r v . i$ ist vollständig.

Beweis: Wir werden zeigen, dass $r I(U)$ besteht. Sonst gäbe es zwei Elemente $e$ und $e_{\alpha}$ mit $r I(E)$. Auf Grund der Annahme gibt es ein $d$ mit $l v . i, r \varepsilon . i$ und $r v . i$, und folglich gibt es ein gewisses $c$ mit $c h^{\prime}=d$.

Aus

und

$$
c\left(e h^{\prime}\right)=(c e) h^{\prime}=c h^{\prime}=d
$$

$$
c\left(e_{\alpha} h^{\prime}\right)=\left(c e_{\alpha}\right) h^{\prime}=c h^{\prime}=d
$$

erhalten wir $e h^{\prime}=h^{\prime}$ und $e_{\alpha} h^{\prime}=h^{\prime}$.

Wegen $l E$ gelten $h_{1} h^{\prime}=e, h_{1}^{\prime} h_{1}=e$ und $h_{1}{ }^{\prime \prime} h_{1}=e_{\alpha}$. Dann bilden wir

und

$$
h^{\prime}=e h^{\prime}=\left(h_{1}^{\prime} h_{1}\right) h^{\prime}=h_{1}^{\prime}\left(h_{1} h^{\prime}\right)=h_{1}^{\prime} e=h_{1}^{\prime}
$$

$$
h^{\prime}=e_{\alpha} h^{\prime}=\left(h_{1}^{\prime \prime} h_{1}\right) h^{\prime}=h_{1}^{\prime \prime}\left(h_{1} h^{\prime}\right)=h_{1}^{\prime \prime} e=h_{1}^{\prime \prime},
$$

woraus $h_{1}^{\prime \prime}=h_{1}^{\prime}$ folgt. Dann gilt $e_{\alpha}=e$, und $r I(U)$ besteht. Hiermit ist das System auf das vollständige System 14) im Abschnitt II zurückgeführt.

\section{§ 4. Die Systeme $A, E, l E . r i, l U . r i, r i(U)$ und $A, E, l E . l i, l U . l i, \operatorname{ri}(U)$}

Wir wollen nun den folgenden Satz beweisen.

Satz 3. Jedes der Systeme $A, E, l E . r i, l U . r i, r i(U)$ und $A, E, l E . l i, l U . l i, r i(U)$ ist vollständig.

Beweis: Ein Element mit den Eigenschaften $l E . l i$ und $l U . l i$ des Systems $A, E, l E . l i, l U . l i, r i(U)$ hat wegen Hilfssatz 4 im Abschnitt III auch die Eigenschaften $l E . r i$ und $l U . r i$. Es gegnügt folglich, die Vollständigkeit des Systems $A, E, l E . r i, l U . r i, r i(U)$ zu zeigen.

Ein Element $e$ mit $l E . r i$ und $l U . r i$ hat wegen Hilfssatz 2 im Abschnitt III auch die Eigenschaft $I(E)$. Für ein beliebiges $a$ gilt $a^{\prime} a \supset e$, und wegen Hilfssatz 1 im Abschnitt III gilt auch $e a^{\prime} \supset a^{\prime}$. Aus

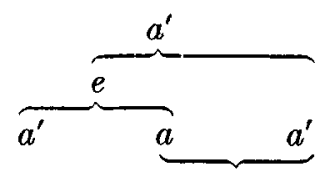

folgen $a a^{\prime} \supset e_{k}$ und $a^{\prime} e_{k} \supset a^{\prime}$. Wegen Hilfssatz $2 \mathrm{im}$ Abschnitt III gilt $e_{k} e_{k} \supset e_{k}$, und wegen $l E . I$ und $l U . I$ gilt $e_{k}^{\prime} e_{k} \supset e$. 
B. SToLr, Weitere Untersuchungen zur Gruppenaxiomatik

Aus

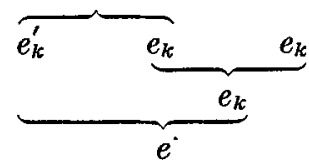

folgen $e_{i} e_{k} \supset e$ und $e_{k}^{\prime} e_{k} \supset e_{i}$. Wegen $l U . I$ gilt $e_{i}=e_{k}^{\prime}$, woraus $e_{k}^{\prime} e_{k} \supset e_{k}^{\prime}$ folgt.

Wir bilden nun

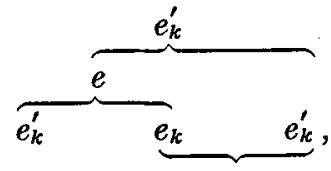

woraus $e_{k} e_{k}^{\prime} \supset e_{h}$ und $e_{k}^{\prime} e_{h} \supset e_{k}^{\prime}$ folgen. Wegen $r i(U)$ gilt $e_{h}=e_{k}$. Hieraus folgt $e_{k} e_{k}^{\prime} \supset e_{k}$. Wegen $r i(U)$ gilt dann $e_{k}^{\prime}=e_{k}$, woraus $e_{k} e_{k} \supset e$ folgt.

Dann bilden wir

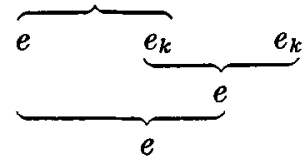

Wegen $l U . I$ gilt dann $e e_{k} \supset e_{k}$. Aus

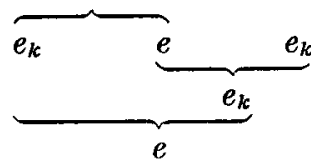

folgt wegen $l U . I e_{k} e \supset e_{k}$. Aus $r i(U)$ folgt $e_{k}=e$, womit gezeigt ist, dass $e$ die Eigenschaft $r E . I$ hat. Hiermit ist das System auf das vollständige System 10) im Abschnitt III zurückgeführt.

\section{§ 5. Die Irreduzibilität der hergeleiteten Systeme}

Es leuchtet unmittelbar ein, dass das erste und das zweite der hergeleiteten Systeme weniger umfassend als 19) im Abschnitt II, und das dritte und das vierte weniger umfassend als 2), 3), 6) und 7) im Abschnitt III sind. In der Tafel der unentschiedenen Systeme des Abschnitts IV fallen 3) und 4) aus, und 2) wird durch die folgenden unentschiedenen Systeme ersetzt.

$$
\begin{aligned}
& A, E, U, l E . I, l U . I, r \varepsilon . I, l i(U), r I(U), \\
& A, E, U, l E . I, l U . I, r v . I, l i(U), r I(U) .
\end{aligned}
$$

Aus der folgenden Tafel geht hervor, dass die vier Systeme irreduzibel sind. 
ARKIV FÖR MATEMATIK. Bd 3 nr 5

\begin{tabular}{|c|c|c|}
\hline Vollständiges System & $\begin{array}{c}\text { Teilsystem, das aus dem } \\
\text { vollständigen System } \\
\text { hervorgeht }\end{array}$ & $\begin{array}{l}\text { Auf Grund folgendes } \\
\text { Satzes des Abschnitts } \\
\text { IV ist das Teilsystem } \\
\text { unvollständig }\end{array}$ \\
\hline$A, E, U, l E . i, l U . i, r \varepsilon . i, r v . i$ & $\begin{array}{l}A, E, l E . i, l U . i, r \varepsilon . i, r v . i \\
A, E, U, l \varepsilon . i, l U . i, r \varepsilon . i, r v . i \\
A, E, U, l E . i, l v . i, r \varepsilon . i, r v . i \\
A, E, U, l E . i, l U . i, r v . i \\
A, E, U, l E . i, l U . i, r \varepsilon . i\end{array}$ & $\begin{array}{ll}\text { Satz } & 9 \\
\text { Satz } & 1 \\
\text { Satz } & 3 \\
\text { Satz } & 5 \\
\text { Satz } & 4\end{array}$ \\
\hline$A, E, l E, U, l v . i, r \varepsilon . i, r v . i$ & $\begin{array}{l}A, l E, U, l v . i, r \varepsilon . i, r v . i \\
A, E, l E . i, U, l v . i, r \varepsilon . i, r v . i \\
A, E, l E, l v . i, r \varepsilon . i, r v . i \\
A, E, l E, U, r \varepsilon . i, r v . i \\
A, E, l E, U, l v . i, r v . i \\
A, E, l E, U, l v . i, r \varepsilon . i\end{array}$ & $\begin{array}{ll}\text { Satz } & 8 \\
\text { Satz } & 3 \\
\text { Satz } & 9 \\
\text { Satz } & 7 \\
\text { Satz } & 5 \\
\text { Satz } & 4\end{array}$ \\
\hline$A, E, l E . r i, l U . r i, r i(U)$ & $\begin{array}{l}A, E, l \varepsilon . r i, l U . r i, r i(U) \\
A, E, l E . r i, l v . r i, r i(U) \\
A, E, l E . i, l U . i, r i(U) \\
A, E, l E . r i, l U . r i, r I(U)\end{array}$ & $\begin{array}{lr}\text { Satz } 1 \\
\text { Satz } 10 \\
\text { Satz } 10 \\
\text { Satz } 6\end{array}$ \\
\hline$A, E, l E . l i, l U . l i, r i(U)$ & $\begin{array}{l}A, E, l \varepsilon . l i, l U . l i, r i(U) \\
A, E, l E . l i, l v . l i, \operatorname{ri}(U) \\
A, E, l E . i, l U . i, \operatorname{ri}(U) \\
A, E, l E . l i, l U . l i, r I(U)\end{array}$ & $\begin{array}{lr}\text { Satz } 1 \\
\text { Satz } 10 \\
\text { Satz } 10 \\
\text { Satz } 6\end{array}$ \\
\hline
\end{tabular}

\section{§ 6. Aufstellung der Axiome $E U A_{S t}$ und EUL $L_{L}$}

In den meisten vollständigen Systemen ist es möglich, die Axiome $A$ und $U$ bzw. $A$ und $E$ durch ein einziges Axiom zu ersetzen. Dadurch erreicht man, dass vollständige Systeme hervorgehen, die weniger Axiome enthalten.

LORENZEN ${ }^{1}$ hat das Problem behandelt, die kürzesten vollständigen Axiomensysteme aufzustellen. Er stellt drei Systeme auf, die nur drei Axiome enthalten. In meiner Dissertation werden die von Lorenzen benutzten Formulierungen des assoziativen Gesetzes mit $U A$ und $E A_{L}$ bezeichnet. In der Dissertation werden noch zwei Formulierungen benutzt, die mit $E A_{H}$ und $E A_{S t}$ bezeichnet worden sind. Weil in der Dissertation nur das Problem behandelt wird, die schwächsten vollständigen Systemen aufzustellen, umfassen diese Systeme mehrere Axiome als die Systeme Lorenzens.

Die früber behandelten Formulierungen des assoziativen Gesetzes enthalten entweder Existenzbedingungen oder Unitätsbedingungen. In dieser Arbeit aber wollen wir zwei Formulierungen aufstellen, die sowohl Existenzbedingungen als Unitätsbedingungen enthalten. Dann wollen wir einige Hilfssätze beweisen, und schliesslich werden wir die Vollständigkeit einiger Systeme zeigen.

Die neuen Axiome werden mit $E U A_{S t}$ und $E U A_{L}$ bezeichnet und in folgender Weise formuliert.

1 LorenzeN, P.: Ein Beitrag zur Gruppenaxiomatik. Math. Z. 49 (1943-44), 313-327. 
B. SToLT, Weitere Untersuchungen zur Gruppenaxiomatik

$\boldsymbol{E U}_{\boldsymbol{S}_{t}:}:$ Wenn $z u$ drei Elementen $a, b$ und $c$ die Verknüpfungen $a b \supset u_{h}$ und $u_{h} c \supset t_{i}$ bestehen, und wenn ein oder mehrere Elemente $v_{1}, v_{2}, \ldots, v_{k}, \ldots$ existieren, die $a v_{1} \supset t_{i}$ erfüllen, gibt es unter diesen Elementen ein $v_{k}$, das $b c \supset v_{k}$ erfüllt.

Wenn $z u$ drei Elementen $a, b$ und $c$ die Verknüpfungen $b c \supset v_{k}$ und $a v_{k} \supset w_{j}$ bestehen, und wenn ein oder mehrere Elemente $u_{1}, u_{2}, \ldots, u_{n}, \ldots$ existieren, die $u_{1} c \supset w_{j}$ erfüllen, gibt es unter diesen Elementen ein $u_{h}$, das $a b \supset u_{h}$ erfüllt.

Wenn zu drei Elementen $a, b$ und $e$ die Verknüpfungen $a b \supset u_{h}, u_{h} c \supset t_{i}$, $b c \supset v_{k}$ und $a v_{k} \supset w_{j}$ bestehen, gilt $t_{i}=w_{j}$.

$\boldsymbol{E U} \boldsymbol{A}_{L}:$ Wenn $z u$ drei Elementen $a, b$ und $c$ die Verknüpfungen $a b \supset u_{h}$ und $u_{h} c \supset t_{i}$ bestehen, bestehen auch $b c \supset v_{k}$ und $a v_{k} \supset t_{i}$. Wenn auch $b c \supset v_{k}$, und $a v_{k} \supset w_{j}$ bestehen, gilt $t_{i}=w_{j}$.

Wenn zu drei Elementen $a, b$ und $c$ die Verknüpfungen $b c \supset v_{k}$ und $a v_{k} \supset w_{j}$ bestehen, bestehen auch $a b \supset u_{h}$ und $u_{h} c \supset w_{j}$. Wenn auch $a b \supset u_{h^{\prime}}$ und $u_{h^{\prime}} c \supset t_{i}$ bestehen, gilt $t_{i}=w_{j}$.

Es leuchtet unmittelbar ein, dass das exste Axiom die logische Summe von $U A$ und $E A_{S t}$ und das zweite Axiom die logische Summe von $U A$ und $E A_{L}$ ist. Darum ist es möglich, gewisse Hilfssätze aus der Dissertation zu benutzen.

Es wird hervorgehoben, dass es möglich ist, in allen vollständigen Systemen des Abschnitts VI $E A_{H}$ durch $E A_{s t}$ zu ersetzen. Die vollständigen Systeme dieser Arbeit, die $E U A_{S t}$ bzw. $E U A_{L}$ enthalten, gehen aus den vollständigen Systemen der Abschnitte VI und VIII hervor, wenn die Axiome $E A_{H}$ und $U$ bzw. $E A_{L}$ und $U$ durch $E U A_{s t}$ bzw. $E U A_{L}$ ersetzt werden. Die Beweise sind aber viel mühsamer.

Wir werden auch die Vollständigkeit dreier Systeme zeigen, die aus den folgenden Systemen hervorgehen.

$$
\begin{aligned}
& E A_{s t}, l E, U, l v . i, r \varepsilon . i, r v . i \\
& E A_{L}, U, l E . l i, l U . l i, r \varepsilon . l i, r v . l i \\
& E A_{L}, U, l E . r i, l U . r i, r \varepsilon . r i, r v . r i .
\end{aligned}
$$

Die Vollständigkeit dieser drei Systeme, die nicht in der Dissertation behandelt sind, folgt aus der Vollständigkeit der entsprechenden Systeme, die EUA $A_{s t} \mathrm{bzw}$. $E U A_{L}$ enthalten.

In den vollständigen Systemen 9) und 13) des Abschnitts VIII ist es möglich, das Axiom $U$ wegzulassen. Darum werden wir uns mit der Vollständigkeit der $E U A_{L}$ enthaltenden entsprechenden Systeme nicht beschäftigen. Die Vollständigkeitsbeweise der Systeme

$$
\begin{aligned}
& E A_{L}, l E . l i, l U . l i, r i(U) \\
& E A_{L}, l E . r i, l U . r i, r i(U)
\end{aligned}
$$

sind mit dem Beweis des Satzes 3 analog.

\section{§ 7. Hilfssätze}

Wir werden nun die folgenden Hilfssätze beweisen.

Hilfssatz 1. Wenn $E U A_{S t}, l E$ und lv.i bestehen, bestehen auch $r I(E)$ und $U$.

Beweis: Der Annahme zufolge besteht $h^{\prime} c \supset d$, wo $d$ die Eigenschaft $l v . i$ hat. Wegen $l E$ gilt $e c \supset c$. Dann erhalten wir 
ARKIV För MATEMATIK. Bd 3 nr 5

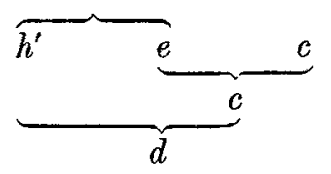

Wegen $E U A_{S t}, l E$ und $l v . i$ gilt $h^{\prime} e \supset h^{\prime} . e$ hat also die Eigenschaft $r i(E)$. Wegen Hilfssatz 6 im Abschnitt $\mathrm{V}$ hat $e$ die Eigenschaft $r I(E)$, und ausserdem besteht $U$, w.z. b.w.

Hilfssatz 2. Wenn $E U A_{S t}, l E, r \varepsilon . i$ und rv.i bestehen, besteht $r I(U)$.

Beweis: Wenn $r I(U)$ nicht bestände, gäbe es zwei Elemente $e, e_{\alpha}$ mit $r I(E)$. Wegen $r \varepsilon . i$ und $r v . i$ gibt es zu zwei Elementen $c$ und $d$ genau ein $h^{\prime}$, das $c h^{\prime} \supset d$ erfüllt. Wir bilden nun
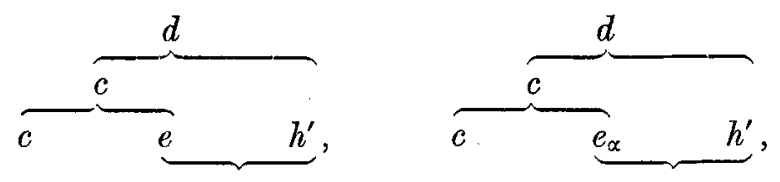

woraus nach $E U A_{s t}, r \varepsilon . i$ und $r v . i e h^{\prime} \supset h^{\prime} b z w . e_{\alpha} h^{\prime} \supset h^{\prime}$ folgen.

Wegen $l E$ gelten $h_{1} h^{\prime} \supset e, h_{1}^{\prime} h_{1} \supset e$ und $h_{1}^{\prime \prime} h_{1} \supset e_{\alpha}$. Dann erhalten wir
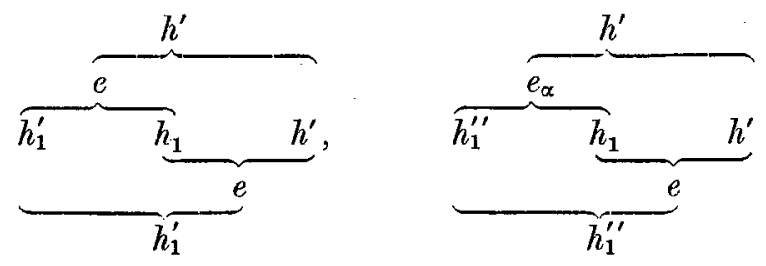

Wegen $E U A_{\text {st }}$ gilt $h_{1}^{\prime}=h^{\prime}=h_{1}^{\prime \prime}$, woraus $h^{\prime} h_{1} \supset e, e_{\alpha}$ folgt.

Schliesslich bilden wir

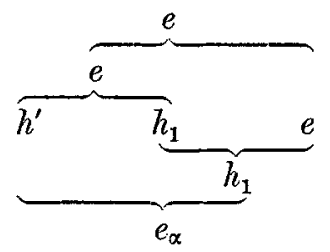

Aus $E U A_{S t}$ folgt $e_{\alpha}=e$, womit der Hilfssatz bewiesen ist.

Hilfssatz 3. Wenn EUA $A_{\text {st }}$ und re.li bestehen, besteht $I(E)$.

Beweis: Der Annahme zufolge bestehen $e c \supset c$ und $c c_{1}^{\prime} \supset e$. Aus

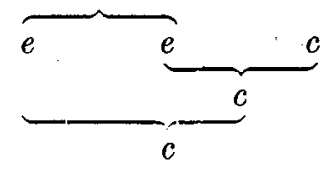


B. StoLt, Weitere Untersuchungen zur Gruppenaxiomatik

folgt wegen $E U A_{S t}$ und $l i(E) e e \supset e_{\alpha}$ und $e_{\alpha} c \supset c$, und aus

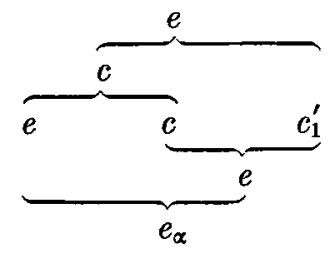

folgt wegen $E U A_{s t} e_{\alpha}=e$, womit der Hilfssatz bewiesen ist.

Hilfssatz 4. Wenn EUA,$l E . I$ und $l U . I$ bestehen, und wenn $e$ ein Element mit $l E . I$ und $l U . I$ und a ein beliebiges Element ist, bestehen $a^{\prime} a \supset e, e a^{\prime} \supset a^{\prime}$, $a a^{\prime} \supset e_{k}, a^{\prime} e_{k} \supset a^{\prime}$ und $e_{k} e_{k} \supset e_{k}$.

Beweis: Wegen Hilfssatz $3 \mathrm{im}$ Abschnitt VIII bestehen $a^{\prime} a \supset e, e a^{\prime} \supset a^{\prime}$, $a a^{\prime} \supset e_{k}$ und $a^{\prime} e_{k} \supset a^{\prime}$, wo $e$ ein Element mit $l E . I$ und $l U . I$ und $a$ ein beliebiges Element ist.

Aus

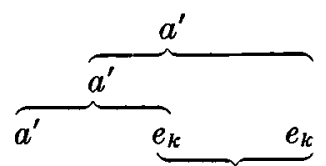

folgt wegen $E U A_{L} e_{k} e_{k} \supset e_{h}$ und $a^{\prime} e_{h} \supset a^{\prime}$. Wir bilden auch

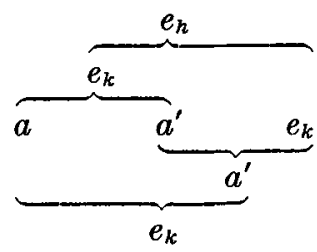

Aus $E U A_{L}$ folgt $e_{h}=e_{k}$, und dann gilt $e_{k} e_{k} \supset e_{k}$, w. z. b.w.

\section{§ 8. Systeme, die $E \boldsymbol{U} \boldsymbol{A}_{S t}$ enthalten}

Wir wollen nun die Vollständigkeit der folgenden Systeme zeigen.

1) $E U A_{s t}, l E, l i(U), r I(U)$

2) $E U A_{S t}, l E, l v . i, r I(U)$

3) $E U A_{s t}, l E, r \varepsilon . l i, r I(U)$

4) $E U A_{S t}, l E, r \varepsilon . i, r v . i, l i(U)$

5) $E U A_{S t}, l E, r \varepsilon . l i, r v . l i$

6) $E U A_{s t}, l E, l v . i, r \varepsilon . i, r v . i$. 
Satz 4. Das System $E U A_{S t}, l E, l i(U)$ und $r I(U)$ ist vollständig.

Beweis: Wegen Hilfssatz 2 im Abschnitt VII besteht ein Element mit $I(E)$, womit das System auf das vollständige System 4) im Abschnitt V zurückgeführt ist.

Satz 5. Das System $E U A_{S t}, l E, l v . i$ und $r I(U)$ ist vollständig.

Beweis: Wegen Hilfssatz 1 bestehen $r I(E)$ und $U$, womit das System auf das vollständige System 4) im Abschnitt II zurückgeführt ist.

Satz 6. Das System $E U A_{S t}, l E$, re.li und $r I(U)$ ist vollständig.

Beweis: Wegen Hilfssatz 3 besteht $I(E)$, womit das System auf das vollständige System 4) im Abschnitt $V$ zurückgeführt ist.

Satz 7. Das System $E U A_{S t}, l E, r \varepsilon . i, r v . i$ und $l i(U)$ ist vollständig.

Beweis: Wegen Hilfssatz 2 im Abschnitt VII besteht $I(E)$, und wegen Hilfssatz 2 besteht $r I(U)$, womit das System auf das vollständige System 4) im Abschnitt $V$ zurückgeführt ist.

Satz 8. Das System $E U A_{S t}, l E$, re.li und ru.li ist vollständig.

Beweis: Wegen Hilfssatz 3 besteht $I(E)$, und wegen Hilfssatz 2 besteht $r I(U)$, womit das System auf das vollständige System 4) im Abschnitt V zurückgeführt ist.

Satz 9. Das System $E U A_{S t}, l E, l v . i, r \varepsilon . i$ und rv.i ist vollständig.

Beweis: Wegen Hilfssatz 1 bestehen $r I(E)$ und $U$, und wegen Hilfssatz 2 besteht $r I(U)$, womit das System auf das vollständige System 4) im Abschnitt II zurückgeführt ist.

\section{§ 9. Systeme, die $E U A_{L}$ enthalten}

Schliesslich werden wir die Vollständigkeit der folgenden Systeme zeigen.

1) $E U A_{L}, l E, l I$

2) $E U A_{L}, l E . r i, r U . r i$

3) $E U A_{L}, l E . l i, l U . l i, I(U)$

4) $E U A_{L}, l E . r i, l U . r i, I(U)$

5) $E U A_{L}, l E . l i, l U . l i, r \varepsilon . l i, r v . l i$

6) $E U A_{L}, l E . r i, l U . r i, r \varepsilon . r i, r v . r i$

7) $E U A_{L}, l E . r I, r I(U)$

8) $E U A_{L}, l E . r I, r \varepsilon . r I, r v . r I$

9) $E U A_{L}, l E . l i, r U . l i, r \varepsilon . l i$

10) $E U A_{L}, l E . l i, r U . l i, l v . l i$.

Das System 1) ist schwächer als die Systeme 4) und 5) Lorenzens und enthält nur zwei Axiome. In der Tafel gibt es zwei Systeme, die drei Axiome enthalten. 
B. STоLT, Weitere Untersuchungen zur Gruppenaxiomatik

Satz 10. Das System EUA $A_{L}, l E . l I$ ist vollständig.

Beweis: Wegen Hilfssatz $1 \mathrm{im}$ Abschnitt $\mathrm{V}$ besteht $U$, womit das System auf das vollständige System 1 im Abschnitt VIII zurückgeführt ist.

Satz 11. Das System EUA $A_{L}$, IE.ri und rU.ri ist vollständig.

Beweis: Wegen Hilfssatz 3 gibt es ein Element mit $l E . I$ und rU.I, und wegen Hilfssatz 2 im Abschnitt VIII besteht $E$. Hiermit ist das System auf das vollständige System 5) im Abschnitt $\mathrm{V}$ zurückgefürt.

Satz 12. Das System EUA $A_{L}, l E . l i, l U . l i$ und $I(U)$ ist vollständig.

Beweis: Der Annahme zufolge gibt es ein Element $e$ mit $l E . l i$ und $l U . l i$, das wegen Hilfssatz 4 im Abschnitt VIII auch $l E$.ri und wegen Hilfssatz 3 sogar $l E . I$ und $l U . I$ hat. Wenn $a$ ein beliebiges Element ist, bestehen wegen Hilfssatz 4 $a^{\prime} a \supset e, a a^{\prime} \supset e_{k}$ und $e_{k} e_{k} \supset e_{k}$. Wegen $I(U)$ gilt $e_{k}=e$. Dann hat $e$ die Eigenschaft rE.I, womit dieses System auf 2) zurückgeführt ist.

Satz 13. Das System EUA $A_{L}, l E . r i, l U . r i$ und $I(U)$ ist wollständig.

Beweis: Wegen Hilfssatz 3 bestehen $l E . I$ und $l U . I$, womit dieses System auf 2) zurückgeführt ist.

Satz 14. Das System $E U A_{L}, l E . l i, l U . l i, r \varepsilon . l i$ und rv.li ist vollständig.

Beweis: Der Annahme zufolge bestehen $e c \supset c$ und $e c^{\prime} \supset e$, wo $e$ die Eigenschaften $l E . l i, l U . l i$, re.li und rv.li hat. Wegen Hilfssatz 3 hat $e$ auch die Eigenschaft $I(E)$, und wenn $a$ ein beliebiges Element ist, bestehen nach Hilfssatz 4 $a^{\prime} a \supset e, a a^{\prime} \supset e_{k}$ und $e_{k} e_{k} \supset e_{k}$. Wir wollen zeigen, dass $e_{k}$ gleich $e$ ist.

Nach $l E . I$ und $l U . I$ gilt $e_{k}^{\prime} e_{k} \supset e$. Aus

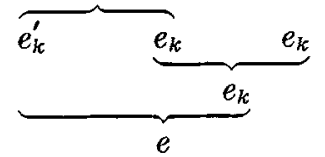

folgt nach $E U A_{L}, e_{k}^{\prime} e_{k} \supset e_{k}^{\prime}$, und aus

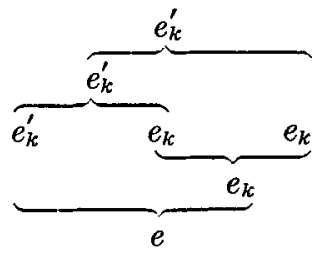

folgt nach $E U A_{L}, e_{k}^{\prime}=e$. Dann gilt $e e_{k} \supset e$. Wir bilden ferner

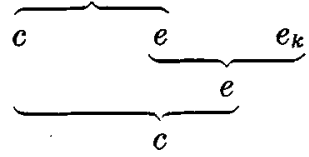

und nach $E U A_{L}$ gelten $c e \supset c_{\alpha}$ und $c_{\alpha} e_{k} \supset c$. Aus 
ARKIV FöR MATEMATIK. Bd 3 nr 5

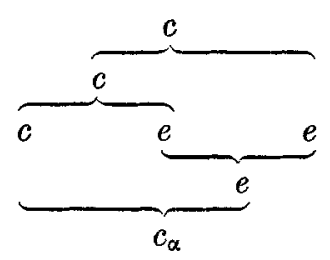

folgt nach $E U A_{L} c_{\alpha}=c$, und dann gilt $c e_{k} \supset c$.

Nach $l E . I$ und $l U . I$ bestehen $c_{\alpha}^{\prime} c \supset e$ und $c_{\beta} c_{\alpha}^{\prime} \supset e$. Aus

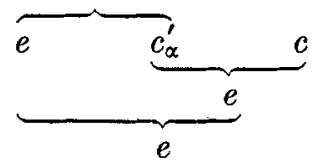

folgt $e c_{\alpha}^{\prime} \supset e_{\alpha}^{\prime}$, aus

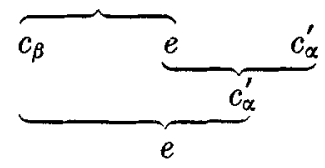

folgt $c_{\beta} e \supset c_{\beta}$, und aus

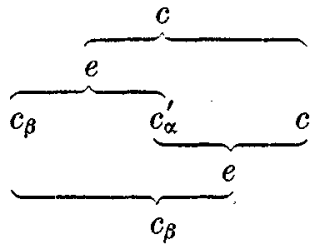

folgt $c_{\beta}=c$. Nach $l U . I$ gilt dann $c^{\prime} c \supset e$. Wegen

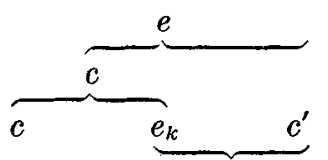

gilt $e_{k} c^{\prime} \supset c^{\prime}$, und wegen

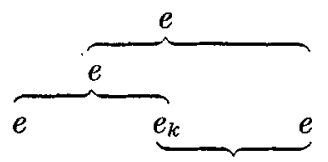

gilt $e_{k} e \supset e_{h}$. Schliesslich bilden wir

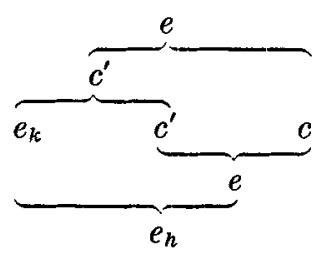




\section{B. stoLt, Weitere Untersuchungen zur Gruppenaxiomatik}

woraus nach $E U A_{L} e_{h}=e$ folgt. Dann gilt $e_{k} e \supset e$, und nach $l U . I$ gilt $e_{k}=e$. $e$ hat also die Eigenschaft $r E . I$, womit das System auf 2) zurückgeführt ist.

Satz 15. Das System EUA $A_{L}, l E . r i, l U . r i, r \varepsilon . r i$ und rv.ri ist vollständig.

Beweis: Wegen Hilfssatz 3 hat ein Element mit $l E . r i, l U . r i, r \varepsilon . r i$ und rv.ri auch die Eigenschaft $I(E)$, womit das System auf das vorige System zurückgeführt ist.

Satz 16. Die Systeme EUA $A_{L}, l E . r I, r I(U)$ und $E U A_{L}, l E . r I, r \varepsilon . r l$, rv.rI sind vollständig.

Beweis: Wegen Hilfssatz 1 im Abschnitt V besteht $U$, womit die Systeme auf die vollständigen Systeme 17) bzw. 18) im Abschnitt VIII zurückgeführt sind.

Satz 17. Die Systeme EUA $A_{L}, l E . l i, r U . l i, r \varepsilon . l i$ und $E U A_{L}, l E . l i, r U . l i, l v . l i$ sind vollständig.

Beweis: Wegen Hilfssatz 3 bzw. Hilfssatz 4 im Abschnitt VIII besteht ein Element mit $l E . I$ und $r U . I$, womit die Systeme auf 2) zurückgeführt sind.

\section{\$ 10. Systeme, die aus höchstens drei Axiomen bestehen}

Schliesslich soll gezeigt werden, dass es nur acht vollständige Systeme gibt, die höchstens drei Axiome enthalten. Dazu ist es notwendig, alle vollständigen Systeme zu bilden, die eines oder mehrere der Axiome $A, E, U$ und höchstens zwei andere Axiome enthalten. Dann wollen wir untersuchen, ob es möglich ist, einige der Axiome $A, E, U$ durch eine gewisse Formulierung des assoziativen Gesetzes zu ersetzen.

Zuerst betrachten wir die unvollständigen Systeme des Abschnitts IV mit Berücksichtigung der Resultate des $\S 5$. Wenn wir aus diesen Systemen Teilsysteme bilden, die eines oder mehrere von $A, E, U$ und höchstens zwei andere Axiome enthalten, erhalten wir nur Systeme, die wegen der Sätze des Abschnitts IV unvollständig sind.

In den Abschnitten II und III gibt es vollständige Systeme, die eines oder mehrere von $A, E, U$ und höchstens zwei andere Axiome enthalten. Aus einigen der übrigen Systeme ist es möglich, solche Systeme zu bilden, indem man zwei Axiome durch ein stärkeres Axiom ersetzt. Es ist möglich, die folgenden vollständigen Systeme zu bilden.
1) $A, l E, U, l I(E)$
5) $A, E, l U, r E . l i$
2) $A, l E, U, r E . i$
6) $A, E, U, l E . l I$
3) $A, l E, U, r U . r i$
7) $A, E, U, l E . r i, l U . r i$
4) $A, E, l U, l E . l I$
8) $A, E, U, l E . r I, r I(U)$.

Die Systeme 1)-6) kommen in der Arbeit Lorenzens vor. 3) -5) sind reduzibel, wenn man die Axiomemenge meiner Dissertation betrachtet. In die von Lorenzen betrachteten Axiomemenge sind sie irreduzibel. Lorenzen hat gezeigt, dass 1)-6). die einzigen $A$ enthaltenden viergliedrigen vollständigen Systeme sind. 
ARKIV FöR MATEMATIK. Bd $3 \mathrm{nr} 5$

Wenn Wir in 1)-8) die Axiome $A, E, U$ durch eines der Axiome $U A, E A_{L}$ oder $E U A_{L}$ ersetzen, erhalten wir die folgenden Systeme.
1) $U A, l E, l I(E)$
5) $E A_{L}, l U, r E . l i$
2) $U A, l E, r E . i$
6) $E U A_{L}, l E . l I$
3) $U A, l E, r U . r i$
7) $E U A_{L}, l E . r i, l U . r i$
4) $E A_{L}, l U, l E . l I$
8) $E U A_{L}, l E . r I, r I(U)$.

Die Vollständigkeit von 1)--5) ist von Lorenzen, die von 6)-8) in der vorliegenden Arbeit bewiesen. Es ist klar, dass diese Systeme die einzigen vollständigen Systeme sind, die höchstens dreigliedrig sind und aus der Menge der betrachteten Axiome gebildet werden können. 